\title{
Role of Radiotherapy in Vulvar Squamous Cell Carcinoma: Review of the Literature
}

\author{
(1) Çağlayan Selenge BEDÜK ESEN, (1) Melis GÜLTEKIN, (1) Ferah YILDIZ
}

Department of Radiation Oncology, Hacettepe University Faculty of Medicine, Ankara-Turkey

\begin{abstract}
SUMMARY
En bloc radical vulvectomy with bilateral inguinofemoral lymph node dissection was the standard approach for patients diagnosed with vulvar cancer, regardless of disease extent in the past. This radical surgical approach was with high rates of morbidity and adverse psychological effects and led to insufficient locoregional control rates in patients with locally advanced-stage disease. In recent years, multimodal treatment approaches have been introduced to decrease treatment-related morbidity and increase the locoregional control rates. Radiotherapy (RT) has a major role in the treatment of vulvar cancer. It has been successfully used as an adjuvant approach in patients with high risk of local recurrence after surgery and as a sole local treatment with concomitant chemotherapy in locally advanced disease. In this current review, we will discuss the rationale of RT and provide up-to-date information about the role of RT in the treatment of vulvar cancer.
\end{abstract}

Keywords: Chemoradiotherapy; radiotherapy; treatment; vulvar cancer.

Copyright $\odot$ 2021, Turkish Society for Radiation Oncology

\section{Introduction}

Vulvar cancer is a less common malignancy than other gynecologic tumors, comprising $0.53 \%$ of all cancers in women, and $5.2 \%$ of all gynecological malignancies.[1] Squamous cell carcinoma (SCC) is the most common histological subtype comprising $80-90 \%$ of all cases. [2] Most patients are diagnosed with localized disease and 5 -year survival rates in these patients are around $87 \%$. The corresponding 5-year survival rates for patients with lymph node (LN) metastasis on the other hand are only $50 \%$.[3]

The International Society for the Study of Vulvovaginal Disease updated the terminology used to characterize vulvar lesions as low-grade squamous intraepithelial lesion or usual-type vulvar intraepithelial neoplasia due to flat condyloma or human papilloma virus (HPV) 6-11 infection, high-grade squamous intraepithelial lesion or uVIN 2-3 due to carcinogenic strains of HPV, and differentiated-type VIN (dVIN) associated with vulvar dermatologic conditions such as lichen sclerosis.[4] uVIN tends to occur in young patients in the third to fifth decades of life, on the contrary, dVIN typically occurs in older patients in the sixth to eighth decades of life.[4] Well-known risk factors for the development of vulvar cancer include increasing age, HPV infection, smoking, multiple sexual partners, and immunosuppression. [5]

Vulvar cancer mainly spreads through direct extension to adjacent structures and the lymphatics, first inguinofemoral followed by pelvic LNs. Prognosis is strongly correlated with LN involvement and depth of invasion $(>5 \mathrm{~mm})$. Positive or close $(<8 \mathrm{~mm})$ surgical margin, International Federation of Gynecology and Obstetrics (FIGO) stage, tumor size, tumor localization, lymphovascular space invasion (LVSI), and presence of HPV infection are also associated with treatment outcomes.[6-8]
Received: August 06, 2021

Accepted: September 04, 2021

Online: September 16, 2021

Accessible online at:

www.onkder.org

OPEN ACCESS This work is licensed under a Creative Commons Attribution-NonCommercial 4.0 International License.
Dr. Çağlayan Selenge BEDÜK ESEN

Hacettepe Üniversitesi Tıp Fakültesi,

Radyasyon Onkolojisi Anabilim Dalı,

Ankara-Turkey

E-mail: selengebedk@gmail.com 
Due to the rarity of vulvar cancer, treatment strategies mainly come from the results of limited number of retrospective studies. Historically, radical surgery including radical vulvectomy and bilateral inguinofemoral LN dissection (IFLND) was the standard surgical approach for patients diagnosed with vulvar cancer, regardless of disease extent. However, due to morbidity, psychological effects of exenterative surgery, and insufficient locoregional control rates in patients with locally advanced-stage disease, multimodal treatment approaches have been developed to decrease treatmentrelated morbidity. Radiotherapy (RT) has a major role in the treatment of vulvar cancer; as a neoadjuvant therapy to improve resectability and provide less radical surgery, as a definitive therapy for an inoperable disease, or as an adjuvant therapy to prevent local-regional recurrences. In this review, we will discuss the rationale of RT in neoadjuvant, definitive, and adjuvant settings.

\section{Treatment of Early-Stage Disease (Stages I-II)}

\section{Surgery}

Historically, en bloc radical vulvectomy with bilateral IFLND referred to as "butterfly resection" was the standard approach for vulvar cancer, regardless of disease extent.[9] However, this exenterative surgery has serious postoperative morbidity and psychological sequelae. IFLND alone has also 21-47\% risk of lymphedema. $[10,11]$ In a Cochrane meta-analysis, it was shown that radical vulvectomy and radical local excision with an at least $1 \mathrm{~cm}$ tumor free margin had similar low rates of recurrence and the triple incision technique was as effective as butterfly resection with less morbidity.[12] Again, in a study comparing triple incision technique to butterfly technique by Van der Velden et al.,[13] no difference was observed in overall survival (OS) and disease-free survival rates, however, triple incision technique was found to be an independent prognostic factor for vulvar recurrences in patients with LN metastasis but not for inguinal/pelvic recurrences. Burke et al.[14] also reported that contralateral groin recurrence was a rare event after unilateral LN dissection in patients with welllateralized tumors. Today, simple or radical partial vulvectomy is recommended for $\mathrm{T} 1$ or small $\mathrm{T} 2(\leq 4 \mathrm{~cm})$ tumors and only ipsilateral inguinofemoral LN evaluation is recommended in case of well-lateralized tumors.[15]

\section{Lymphatic Staging and Role of RT as Alternative to IFLND}

Vulvar cancer spreads from the vulva to the ipsilateral superficial inguinofemoral LNs, followed by deep in- guinofemoral LNs, and then to the pelvic LNs.[16] Involvement of contralateral inguinofemoral LNs or pelvic LNs without the involvement of ipsilateral LNs is a rare event, however, the risk of contralateral LN metastases increase with the tumors approaching or crossing the midline.[17] The frequency of inguinal LN metastases in surgical series ranges from $6 \%$ to $50 \%$ depending on the extent of the primary disease. $[18,19]$ Homesley et al.[20] found that greater tumor thickness, older age, LVSI, and tumor grade were risk factors for LN involvement. In one study, perineural invasion was also shown to be a strong risk factor for LN metastasis.[19] Ipsilateral LN evaluation is recommended for well-lateralized ( $\geq 2 \mathrm{~cm}$ from the midline) tumors deeper than $1 \mathrm{~mm}$ and contralateral LN evaluation should be added for tumors approaching or crossing the midline.[15]

For lymphatic staging, sentinel LN biopsy (SLNB) in clinically LN-negative early-stage vulvar cancer has been tested in GROINSS-V-I and GOG 173 trials as a conservative approach instead of IFLND.[21-23] SLNB was found to be safe and effective with less lymphedema and lower groin recurrence rates in patients with tumor-free sentinel LNs.[22,23] The GOG 173 trial showed that the false-negative rate of SLNB was only $2 \%$ with tumors $<4 \mathrm{~cm}$ and it was $7.4 \%$ with tumors $\geq 4 \mathrm{~cm}$.[23] Today, SLNB is accepted as an alternative option to IFLND for lymphatic staging in T1b or T2 tumors.[15]

GROINSS-V II/GOG 270 trial investigated whether RT was a safe alternative for IFLND in vulvar cancer patients with a metastatic sentinel LN.[24,25] In this prospective multicenter Phase II trial, patients were included with early-stage SCC of the vulva (diameter $<4 \mathrm{~cm}$ ) without suspicious LNs at imaging, who had primary surgery with SLNB. In case of a metastatic sentinel LN in any size, 50 Gy conventionally fractionated RT was given to the groin(s). In case of a negative sentinel LN, patients were followed-up for $\geq 2$ years. From December 2005 to October 2016, 1552 eligible patients were registered. Sentinel LN metastasis was found in 21\% (324/1552) of patients. After 54 months of inclusion, the trial was stopped due to increased risk of groin recurrence $(2.1 \%$ vs. $20 \%)$ in case of sentinel $\mathrm{LN}>2 \mathrm{~mm}$ and/or with extracapsular extension (ECE).[25] The treatment protocol was amended with patients with micrometastasis $(\leq 2 \mathrm{~mm})$ in sentinel $\mathrm{LN}$ receiving RT from then on, and those with $>2 \mathrm{~mm}$ in sentinel LN undergoing IFLND (with adjuvant RT in case of $>1 \mathrm{LN}$ and/or ECE). Isolated groin recurrences in patients with a sentinel LN micrometastasis $(\leq 2$ 
$\mathrm{mm}$ ) decreased to $3.2 \%$ with this new treatment protocol and the combination of RT with SLNB was associated with minimal toxicity: $4.2 \%$ Grade 3 toxicity and no Grade 4 or 5 toxicity.[25] This study showed that RT to the groin is a safe alternative to IFLND with minimal toxicity in patients with micrometastasis in sentinel LNs. However, RT alone with a dose of 50 Gy is not sufficient for macrometastases. The ongoing GROINSS-V-III trial investigates the effectiveness and safety of chemoradiotherapy (CRT) (56 Gy to the involved site combined with weekly $40 \mathrm{mg} / \mathrm{m}^{2}$ cisplatin) in patients with macrometastases in sentinel LNs.

\section{Adjuvant Treatment}

Adjuvant RT is commonly used to reduce the risk of recurrences in vulvar cancer patients with high-risk features. The indications of adjuvant RT should be considered for primary tumor site and lymphatics separately. In patients with negative LNs, indication for primary tumor site RT depends on the adverse primary tumor risk factors. The most accepted primary tumor risk factor for recurrence is positive or closes surgical margins. [26,27] Ignatov et al.[27] observed that 5-year OS was higher $(67.6 \%$ vs. $29 \%)$ in patients with positive or close $(<1 \mathrm{~cm})$ surgical margin who received adjuvant RT than those who did not. Viswanathan et al.[26] also reported that vulvar relapses increased in patients with positive or close $(<5 \mathrm{~mm})$ surgical margins, and adjuvant RT reduced local recurrences from $35 \%$ to $21 \%$ in those patients. Two retrospective studies also reported that none of the patients with surgical margin larger than 8 $\mathrm{mm}$ had a vulvar recurrence, but $21-48 \%$ of those with a surgical margin less than $8 \mathrm{~mm}$ had local recurrences. $[28,29]$ Based on the retrospective series, RT has been generally recommended for patients with $<8 \mathrm{~mm}$ surgical margins. However, the need for a minimal margin of $8 \mathrm{~mm}$ was not confirmed in the AGO-CaRE database and recent retrospective studies.[30-32] The definition of close surgical margin remains unclear, but the cutoff value of $8 \mathrm{~mm}$ for surgical margin is widely accepted. Although, $5 \mathrm{~mm}$ surgical margin is accepted adequate by some. All patients with close or positive surgical margins initially should be evaluated for reexcision. In cases whom surgery cannot be performed (tumor close to urethra, clitoris, anal sphincter, etc.), RT should be considered. The recent National Comprehensive Cancer Network (NCCN) recommends adjuvant RT for patients with positive or close surgical margins and accepts $5-8 \mathrm{~mm}$ as the definition of close surgical margin.[15]

The second important risk factor for the recommendation of adjuvant RT to primary tumor site is the depth of tumor invasion. Depth of invasion correlates not only with local recurrences but also with the risk of LN metastasis and subsequently survival rates.[33] There are several studies investigating the cutoff value for depth of invasion to predict recurrences.[6,34] Bogani et al.[34] showed increased rate of local recurrences in patients with a stromal invasion $>2 \mathrm{~mm}$ than those with $\leq 2 \mathrm{~mm}$. Zapardiel et al.[6] also found the stromal invasion of more than $5 \mathrm{~mm}$ as a poor prognostic factor for recurrence and OS. In the light of these findings, adjuvant RT for primary tumor site in patients with tumor thickness $>5 \mathrm{~mm}$ is widely recommended.[15] Besides that, NCCN also recommends adjuvant RT to the primary tumor site for tumors with LVSI, large tumors $(>4 \mathrm{~cm})$, and diffuse/spray pattern of invasion, since several studies demonstrated these features as adverse risk factors for recurrence.[15,28,35]

LN involvement is one of the most important prognostic factors in vulvar cancer. Farias-Eisner et al.[36] reported that 5-year OS rates were $45 \%$ and $98 \%$ in patients with and without LN metastasis, respectively. Gynecologic Oncology Group (GOG) 37 trial compared pelvic LN dissection to pelvic RT after radical vulvectomy and IFLND in patients with pathologically positive inguinofemoral LNs.[37] Within 74 months of follow-up, patients receiving RT had improved 6-year OS rate $(41 \%$ vs. $51 \%)$ and decreased 6 -year cancerspecific death and groin recurrence rates (51\% vs. $29 \%$ and $24 \%$ vs. 5\%, respectively).[37] The subgroup of patients who got the most benefit with adjuvant RT was those with macrometastasis, ECE, or $\geq 2 \mathrm{LN}$ metastases. Although adjuvant RT in patients with $\geq 2$ LN metastases or ECE is not debatable, there are conflicting results in the literature regarding the role of adjuvant RT in patients with only one LN metastasis.[38-41] The AGO-CaRE-1 study was conducted to investigate the role of adjuvant RT in patients with positive LNs and adjuvant RT was administered in $41 \%$ of patients with LN metastasis[38] and revealed that adjuvant RT improved OS (57.7\% vs. $51.4 \%)$ and progression-free survival $(39.6 \%$ vs. $25.9 \%)$ in the whole cohort. However, this improvement was significant especially for patients with $\geq 2$ positive LNs, without any significant survival benefit for patients with only one LN metastasis.[38] Van der Velden et al.[39] also recently supported omitting RT to the groin in patients with a single occult intracapsular LN metastasis due to the low risk of groin recurrence and the excellent groin recurrence-free survival without adjuvant RT. On the other hand, the Surveillance, Epidemiology, and End Results (SEER) database analysis revealed that adjuvant RT 
statistically significantly improved survival, especially in patients with resected LNs less than 12 and single LN metastasis. [40] Of the 490 patients with Stage III, node-positive vulvar cancer in this SEER analysis had a single positive inguinal LN. The median number of LNs resected was 13 (range: 1-34). Patients who received adjuvant RT had a 5-year disease-specific survival of $77.0 \%$ compared to $61.2 \%$ in those without RT $(\mathrm{P}=0.02)$. After stratifying the study group based on the extent of lymphadenectomy, 5-year survival rates with RT were $76.6 \%$ in patients with $\leq 12 \mathrm{LNs}$ removed which was statistically significantly higher than those without RT in whom survival rate was $55.1 \%$. In those with more than 12 nodes resected, RT increased the survival from $66.7 \%$ to $77.3 \%$, though this difference was not statistically significant.[40] Serre et al.[41] also reported that 5-year OS rates were similar in patients with one intracapsular LN metastasis, one extracapsular LN metastasis, and two LN metastases, furthermore, they suggested adjuvant RT to patients with LN metastasis, irrespective of the number of involved LNs in case of LVSI.

There are no randomized data comparing adjuvant RT with CRT. Gill et al.[42] supported the addition of chemotherapy in adjuvant setting using the data from National Cancer Data Base (NCDB). Among 1797 vulvar cancer patients with positive LNs, $26.3 \%$ received adjuvant chemotherapy in addition to adjuvant RT and 76.6\% had 1-3 involved LNs. Adjuvant chemotherapy increased OS (44 vs. 29.7 months) and reduced the risk of death ( $\mathrm{HR}=0.62$; 95\% CI, 0.48-0.79; $\mathrm{P}<0.001)$. [42] Based on all these retrospective studies, adjuvant RT is strongly recommended for patients with $\mathrm{LN}$ metastasis in SLNB or IFLND and CRT is strongly recommended for patients with $\geq 2$ positive LNs or a single $L N$ with $>2$ $\mathrm{mm}$ metastasis after IFLND.[15] Based on limited experience, the most commonly used concurrent cytotoxic agent is weekly cisplatin with a dose of $40 \mathrm{mg} / \mathrm{m}^{2}$.[43]

\section{Treatment of Locally Advanced-Stage Disease (Stages III-IVA)}

The locally advanced disease is defined as patients with tumors extending to the adjacent structures (large [ $\geq 4$ $\mathrm{cm}$ ] T2 and all T3 tumors) or clinically positive groin LNs (FIGO Stages III-IVA). In this phase, the disease is considered unresectable or needs exenterative surgery which leads to significant post-operative complications and decreased quality of life. Thus, a multimodality treatment has been explored to reduce surgery-related morbidity and improve organ preservation rates.[44] RT is commonly used in locally advanced-stage disease as neoadjuvant or definitive treatment.

\section{Neoadjuvant Treatment}

Neoadjuvant RT/CRT can be used in patients with locally advanced-stage disease who are considered inoperable or required extensive surgery to improve the resectability rate and reduce the extent of the surgery. Pre-operative RT in locally advanced disease was first shown to result in tumor debulking and reduce the extent of surgery in a landmark study by Boronow. [45] In that particular study, 5-year OS rate was $75.6 \%$ and pathologically complete response (CR) rate was $40 \%$. [45] There is no randomized study in the literature comparing concurrent CRT with RT alone in locally advanced vulvar cancer, however, concurrent weekly cisplatin or 5-FU alone or in combination is commonly used in locally advanced disease as a radiosensitizer based on Phase III trials which showed the benefit of CRT compared to RT alone in cervical and anal cancers.[46,47] In the GOG 101 study, 73 vulvar cancer patients with unresectable T3-4 tumors received preoperative 47.6 Gy RT in 28 fractions to the pelvis and the inguinofemoral LNs with concurrent cisplatin and 5-FU.[48] The clinical and pathological CR rates in that study were $48 \%$ and $31 \%$, respectively. Only $2.8 \%$ of patients remained unresectable after CRT.[48] Another GOG study which accrued 46 vulvar cancer patients with unresectable N2-3 disease reported CR rates of $41 \%$ in LNs after the same CRT protocol with the GOG 101 trial. [49] In this study, local control rates for LNs and primary tumor were $97 \%$ and $76 \%$, respectively. Although local control and resectability rates were acceptable in these studies, a RT dose of $47.6 \mathrm{~Gy}$ was not sufficient for macroscopic disease. Therefore, GOG 205 trial investigated the effect of increasing RT dose to $57.6 \mathrm{~Gy}$ in 32 fractions with concurrent weekly cisplatin followed by surgery in patients with unresectable T3-4 disease.[50] The authors reported that $69 \%$ of all patients completed the treatment and clinical and pathological CR rates increased to $64 \%$ and $78 \%$, respectively.[50]

\section{Definitive Treatment}

Based on studies evaluating the effectiveness of neoadjuvant RT/CRT, high pathologic CR rates led to a growing acceptance of omitting surgery in locally advanced tumors in case of clinical CR. In the recent NCDB analysis, 2046 patients with locally advanced vulvar cancer who were treated with either definitive RT/CRT or 
pre-operative RT/CRT were analyzed.[51] Within 21.9 months follow-up, 3-year OS was significantly higher ( $57.1 \%$ vs. $41.7 \%$ ) in pre-operative RT/CRT group than primary RT/CRT group.[51] However, on multivariate analysis, OS associated with primary RT/CRT with doses more than 55 Gy was not significantly different from RT/CRT and surgery, and the use of concurrent chemotherapy improved OS of primary RT with doses more than $55 \mathrm{~Gy}$ compared with CRT and surgery. The results of an ongoing GOG 279 trial investigating the role of definitive CRT (a dose of 64 Gy with concurrent cisplatin and gemcitabine) in T2-3, N0-3 vulvar cancer patients are pending. For patients with locally advanced-stage disease who underwent neoadjuvant or definitive RT/CRT, it is recommended to clinically evaluate the patients and perform biopsy within the first 3 months after completion of RT/CRT.[15] If the patient has clinically and pathologically CR, observation is the surveillance policy, but if there is a residual tumor or LN metastasis, surgery or a boost dose of RT should be considered.[15]

\section{RT}

\section{Simulation}

Computed tomography (CT) simulation is critical for appropriate treatment planning in vulvar cancer. A "frog leg" position in a vacuum-evacuated device is generally preferred to spare the skin in the medial thigh. Full bladder and empty rectum are preferred during simulation and treatment to minimize the dose to the small bowel. Internal target volume should be generated in case of locally advanced disease, especially when vagina, urethra, and/or anus are involved. Bolus over the groin is not routinely recommended, but it should be used for large, superficial LNs, or in case of skin involvement.[43] If bolus is used, bolus should be placed over scars with a margin of at least $3 \mathrm{~cm}$. At the time of simulation, patients should undergo scanning both with and without bolus to avoid treatment interruption in case that patients may develop a brisk skin reaction and treatment can be continued without bolus material.[43]

\section{Target Volume Delineation}

Recently, consensus guideline has been published for target delineation and treatment planning in vulvar cancer.[43] Magnetic resonance imaging (MRI) may be useful for delineating the extent of the tumor in definitive treatment. The GTV should encompass all gross lesions according to clinical examination and radiological images. The entire vulva should be included in the CTV, and CTV should be generated with a $1 \mathrm{~cm}$ margin to GTV if GTV extends beyond the vulva. If the primary tumor involves the vagina, $3 \mathrm{~cm}$ margins to GTV should be included in the CTV and the entire vaginal length should be included in the CTV in case of any uncertainty about the proximal extent of the vaginal extension. If the primary vulvar lesion involves the anus, bladder, or urethra, gross disease plus at least 2 $\mathrm{cm}$ of anus, bladder, or urethra should be included in the CTV. Satellite lesions, tumors with extensive LVSI, or dermal lymphatic invasion should be included with extra margins in the CTV. In post-operative settings, CTV should cover the entire operative bed with a margin of $2 \mathrm{~cm}$. CTV to PTV margin should be $0.7-1.0 \mathrm{~cm}$ depending on the patient stability procedure or the treatment technique.

The GTV for LNs may be defined on either MRI or CT and encompasses all gross LNs. Nodal CTV should encompass nodal GTV. If the primary tumor involves the distal vagina, bilateral external iliac, internal iliac, obturator, and inguinofemoral LNs should be included in the nodal CTV. Presacral LNs should also be included in case of involvement of proximal half of the posterior vaginal wall. The inguinofemoral LNs are defined superiorly from where the external iliac artery leaves the bony pelvis to become the femoral artery. The inferior border is $2 \mathrm{~cm}$ below the saphenofemoral junction; however, another commonly used landmark is the level of the lesser trochanter. The ideal perivascular or radial margin (a margin that incorporates the location of all potential inguinal LNs) is not known. Based on a recent study, anteromedial $\geq 35 \mathrm{~mm}$, anterior $\geq 23 \mathrm{~mm}$, anterolateral $\geq 25 \mathrm{~mm}$, and medial $\geq 22 \mathrm{~mm}$ margins to inguinofemoral vessels are recommended to obtain nodal CTV.[52] LN recurrence is not seen posterior or lateral to femoral vessels, thus addition of margins to the vessels in those regions is not recommended. Furthermore, if extensive lymphadenopathy or ECE is noted, covering the entire operative bed should be considered.

\section{RT Dose}

Dose recommendations are mainly based on these consensus guideline and GOG 205 trial protocol. $[15,43,50]$ Definitive CRT is the standard treatment in locally advanced vulvar cancer. A boost dose should be given in the gross disease at the primary site to improve local control and the recommended dose range is $60 \mathrm{~Gy}$ $>70 \mathrm{~Gy}$. In post-operative cases, a minimum equivalent 
dose of 45-50 Gy is recommended for clear margins and 54-60 Gy for close or positive margins. Viswanathan et al.[26] demonstrated an increase of vulvar recurrence rate in vulvar cancer patients with close or positive surgical margin and a minimum dose of 56 Gy decreased recurrence rate from $35 \%$ to $21 \%$ in patients with $0-5$ $\mathrm{mm}$ of surgical margin. A NCDB data evaluating 3075 vulvar cancer patients with positive surgical margin also reported the best survival rate with 54-59.9 Gy. [53] A similar approach is applied for LNs and clinically and/or radiographically uninvolved inguinofemoral LNs should be treated to a "microscopic dose" of 4550 Gy. Positive inguinofemoral LNs without ECE after IFLND should be treated to a dose of 50-55 Gy, and 54$64 \mathrm{~Gy}$ is recommended for LNs with ECE. Minimum of 60-70 Gy should be delivered to inguinofemoral LNs for gross residual or unresectable disease. Dose recommendations and dose constraints for organs at risk are detailed in Tables 1 and 2, respectively.

\section{RT Technique}

RT has a major role in the curative treatment of vulvar cancer. Intensity-modulated RT (IMRT) provides increased tumor control and reduces normal tissue complications. [54] Beriwal et al.[55] comparing threedimensional conformal RT (3DCRT) plans with IMRT

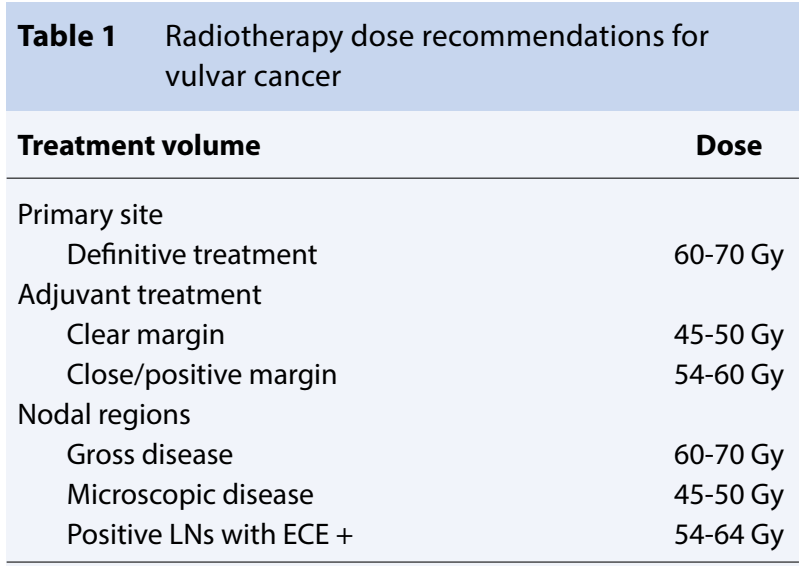

LN: Lymph node; ECE: Extracapsular extension; Gy: Gray

Table 2 Radiotherapy dose constraints for organs at risk

\begin{tabular}{ll} 
Organs at risk & Recommendations \\
\hline Bowel & V40 $<30 \%$ V $45<30 \%$ (minor deviation) \\
Bladder & V45 $<35 \%$ V $50<35 \%$ (minor deviation) \\
$\begin{array}{l}\text { Femoral head } \\
\text { and neck }\end{array}$ & V $30<50 \%$, V $40<35 \%$, and V44 $<5 \%$ \\
Rectum & V45 $<60 \%$ \\
\hline
\end{tabular}

plans in 15 vulvar cancer patients demonstrated that mean bowel, bladder, and rectum doses were significantly lower with IMRT plans. A few studies investigating the role of CRT in the treatment of vulvar cancer used IMRT as a RT technique and reported that IMRT with concurrent chemotherapy is well tolerated, with good pathologic response rates and clinical outcomes.[56-58] Bloemers et al.[59] comparing 3DCRT, sequential IMRT, IMRT with simultaneous integrated boost (SIB), and IMRT with dose-escalated (increased) SIB also reported that all IMRT techniques have lower mean doses in organs at risk than 3DCRT techniques without compromising the dosimetric coverage of the target. The results of the ongoing GOG 279 trial using the IMRT technique are pending. Based on these findings in the literature, IMRT is recommended for the treatment of vulvar cancer. The definitive treatment planning belongs to a patient with Stage IB vulvar cancer is shown in Figure 1.

\section{Conclusion}

The primary aim of the treatment of vulvar cancer is to obtain the best oncological outcomes with the least morbidity. Based on large retrospective and limited number of prospective studies, surgery is preferred treatment option for early-stage vulvar cancer and concurrent CRT is the standard treatment for locally advanced disease. Advances in RT technology and improvements in systemic treatments also show promise in the treatment of vulvar cancers. However, because of their rarity, multicentric studies should be designed.

Peer-review: Externally peer-reviewed.

Conflict of Interest: I have no conflict of interest.

Financial Support: I have no financial support.

\section{References}

1. Siegel RL, Miller KD, Fuchs HE, Jemal A. Cancer statistics, 2021. CA Cancer J Clin 2021;71(1):7-33.

2. Schuurman MS, van den Einden LC, Massuger LF, Kiemeney LA, van der Aa MA, de Hullu JA. Trends in incidence and survival of Dutch women with vulvar squamous cell carcinoma. Eur J Cancer 2013;49:387280.

3. Bethesda M. SEER Cancer Statistics Factsheets: Vulvar Cancer. Tamil Nadu: NIH; 2021.

4. Bornstein J, Bogliatto F, Haefner HK, Stockdale CK, Preti M, Bohl TG, et al. The 2015 international society 


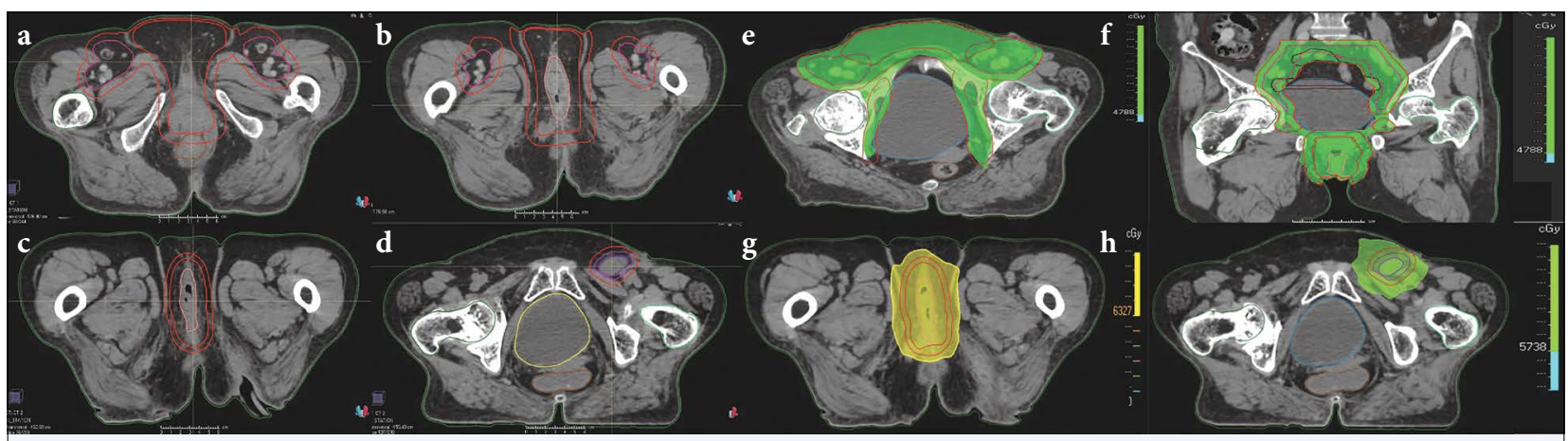

Fig. 1. The treatment planning belongs to a patient with Stage IB vulvar carcinoma who received 50.4 Gy in 28 fractions to pelvic and inguinofemoral lymphatics, a boost dose of $10 \mathrm{~Gy}$ in five fractions to inguinal gross lymph node, and a boost dose of $16.2 \mathrm{~Gy}$ in nine fractions to the primary vulvar lesion with concurrent weekly cisplatin. (a, b) Axial images of computed tomography showing delineation of inguinofemoral lymphatics and the vulva for the first phase of the treatment. ( $c, d$ ) Axial images of computed tomography showing delineation of the primary vulvar lesion and inguinal gross lymph node metastasis for the second phase of the treatment, respectively. (e, f) Axial and coronal images of computed tomography showing dose color wash of the first phase of the treatment. Green isodose line shows $95 \%$ dose color wash of the treatment planning for a total dose of $50.4 \mathrm{~Gy}$. (g, h) Axial images of computed tomography showing dose color wash of the second phase of the treatment. Yellow isodose line shows $95 \%$ dose color wash of the treatment planning for a total dose of 66.6 Gy and green isodose line shows $95 \%$ dose color wash of the treatment planning for a total dose of $60.4 \mathrm{~Gy}$.

Gy: Gray.

for the study of vulvovaginal disease (ISSVD) terminology of vulvar squamous intraepithelial lesions. J Low Genit Tract Dis 2016;20(2):11-4.

5. Madsen BS, Jensen HL, van den Brule AJ, Wohlfahrt J, Frisch M. Risk factors for invasive squamous cell carcinoma of the vulva and vagina--populationbased case-control study in Denmark. Int J Cancer 2008;122(12):2827-34.

6. Zapardiel I, Iacoponi S, Coronado PJ, Zalewski K, Chen F, Fotopoulou C, et al. Prognostic factors in patients with vulvar cancer: The VULCAN study. Int J Gynecol Cancer 2020;30(9):1285-91.

7. Yap J, O’Neill D, Nagenthiran S, Dawson CW, Luesley DM. Current insights into the aetiology, pathobiology, and management of local disease recurrence in squamous cell carcinoma of the vulva. BJOG 2017;124(6):946-54.

8. Cheng X, Zang R, Wu X, Li Z, Cai S, Zhang Z. Recurrence patterns and prognostic factors in Chinese patients with squamous cell carcinoma of the vulva treated with primary surgery. Int J Gynecol Cancer 2009;19(1):158-62.

9. Way S. Carcinoma of the vulva. Am J Obstet Gynecol 1960;79:692-7.

10. Soliman AA, Heubner M, Kimmig R, Wimberger P. Morbidity of inguinofemoral lymphadenectomy in vulval cancer. ScientificWorldJournal 2012;2012:341253.

11. Rouzier R, Haddad B, Dubernard G, Dubois P, Paniel $\mathrm{BJ}$. Inguinofemoral dissection for carcinoma of the vulva: Effect of modifications of extent and technique on morbidity and survival. J Am Coll Surg 2003;196(3):442-50.

12. Ansink A, van der Velden J. Surgical interventions for early squamous cell carcinoma of the vulva. Cochrane Database Syst Rev 2000;2000(2):CD002036.

13. van der Velden J, Schilthuis MS, Hyde SE, Ten Kate FJ, Burger MP. Squamous cell cancer of the vulva with occult lymph node metastases in the groin: The impact of surgical technique on recurrence pattern and survival. Int J Gynecol Cancer 2004;14(4):633-8.

14. Burke TW, Levenback C, Coleman RL, Morris M, Silva EG, Gershenson DM. Surgical therapy of T1 and T2 vulvar carcinoma: Further experience with radical wide excision and selective inguinal lymphadenectomy. Gynecol Oncol 1995;57(2):215-20.

15. National Comprehensive Cancer Network. NCCN Guidelines Version 3.2021 Vulvar Cancer (Squamous Cell Carcinoma). United States: National Comprehensive Cancer Network; 2021.

16. Jolly S, Soni P, Gaffney DK, Biagioli M, Elshaikh MA, Jhingran A, et al. ACR appropriateness criteria(R) adjuvant therapy in vulvar cancer. Oncology (Williston Park) 2015;29(11):867-72.

17. Iversen T, Aas M. Lymph drainage from the vulva. Gynecol Oncol 1983;16(2):179-89.

18. Sedlis A, Homesley H, Bundy BN, Marshall R, Yordan $\mathrm{E}$, Hacker $\mathrm{N}$, et al. Positive groin lymph nodes in superficial squamous cell vulvar cancer. A gyne- 
cologic oncology group study. Am J Obstet Gynecol 1987;156(5):1159-64.

19. Rutledge F, Smith JP, Franklin EW. Carcinoma of the vulva. Am J Obstet Gynecol 1970;106:1117-30.

20. Homesley HD, Bundy BN, Sedlis A, Yordan E, Berek JS, Jahshan A, et al. Prognostic factors for groin node metastasis in squamous cell carcinoma of the vulva (a Gynecologic Oncology Group study). Gynecol Oncol 1993;49(3):279-83.

21. Van der Zee AG, Oonk MH, De Hullu JA, Ansink AC, Vergote I, Verheijen RH, et al. Sentinel node dissection is safe in the treatment of early-stage vulvar cancer. J Clin Oncol 2008;26(6):884-9.

22. Te Grootenhuis NC, van der Zee AG, van Doorn HC, van der Velden J, Vergote I, Zanagnolo V, et al. Sentinel nodes in vulvar cancer: Long-term follow-up of the GROningen INternational Study on Sentinel nodes in Vulvar cancer (GROINSS-V) I. Gynecol Oncol 2016;140(1):8-14.

23. Levenback CF, Ali S, Coleman RL, Gold MA, Fowler JM, Judson PL, et al. Lymphatic mapping and sentinel lymph node biopsy in women with squamous cell carcinoma of the vulva: A gynecologic oncology group study. J Clin Oncol 2012;30(31):3786-91.

24. Brian Slomovitz MO, Monk BJ, Van Driel WJ, Cibula D, DiSilvestro PA, Covens AL, et al. Validation of sentinel lymph biopsy in patients with early stage vulvar cancer: A prospective trial of 1552 women (GROINSV2/ GOG270). In: Annual Meeting on Women's Cancer. Vol. 159. Toronto: Gynecologic Oncology; 2020.

25. van Der Zee AL, de Hullu JA, van Dorst E, Hermans R, Nunns D, Cibula D, et al. Radiotherapy as an alternative treatment for inguinofemoral lymphadenectomy in vulvar cancer patients with a metastatic sentinel node: Results of groinss-v II. In: Annual Meeting on Women's Cancer. Vol. 159. Toronto: Gynecologic Oncology; 2020.

26. Viswanathan AN, Pinto AP, Schultz D, Berkowitz R, Crum CP. Relationship of margin status and radiation dose to recurrence in post-operative vulvar carcinoma. Gynecol Oncol 2013;130(3):545-9.

27. Ignatov T, Eggemann $H$, Burger E, Costa SD, Ignatov A. Adjuvant radiotherapy for vulvar cancer with close or positive surgical margins. J Cancer Res Clin Oncol 2016;142(2):489-95.

28. Heaps JM, Fu YS, Montz FJ, Hacker NF, Berek JS. Surgical-pathologic variables predictive of local recurrence in squamous cell carcinoma of the vulva. Gynecol Oncol 1990;38(3):309-14.

29. Chan JK, Sugiyama V, Pham H, Gu M, Rutgers J, Osann $\mathrm{K}$, et al. Margin distance and other clinico-pathologic prognostic factors in vulvar carcinoma: A multivariate analysis. Gynecol Oncol 2007;104(3):636-41.

30. Woelber L, Griebel LF, Eulenburg C, Sehouli J, Jueckstock J, Hilpert F, et al. Role of tumour-free margin distance for loco-regional control in vulvar cancer-a subset analysis of the arbeitsgemeinschaft gynakologische onkologie CaRE-1 multicenter study. Eur J Cancer 2016;69:180-8.

31. Woelber L, Choschzick M, Eulenburg C, Hager M, Jaenicke F, Gieseking F, et al. Prognostic value of pathological resection margin distance in squamous cell cancer of the vulva. Ann Surg Oncol 2011;18(13):3811-8.

32. Groenen SM, Timmers PJ, Burger CW. Recurrence rate in vulvar carcinoma in relation to pathological margin distance. Int J Gynecol Cancer 2010;20(5):869-73.

33. Yoonessi M, Goodell T, Satchidanand S, Fett W, Solis F. Microinvasive squamous carcinoma of the vulva. J Surg Oncol 1983;24:315-21.

34. Bogani G, Cromi A, Serati M, Uccella S, Donato VD, Casarin J, et al. Predictors and patterns of local, regional, and distant failure in squamous cell carcinoma of the vulva. Am J Clin Oncol 2017;40(3):235-40.

35. Te Grootenhuis NC, Pouwer AW, de Bock GH, Hollema H, Bulten J, van der Zee AG, et al. Prognostic factors for local recurrence of squamous cell carcinoma of the vulva: A systematic review. Gynecol Oncol 2018;148(3):622-31.

36. Farias-Eisner R, Cirisano FD, Grouse D, Leuchter RS, Karlan BY, Lagasse LD, et al. Conservative and individualized surgery for early squamous carcinoma of the vulva: The treatment of choice for stage I and II (T12N0-1M0) disease. Gynecol Oncol 1994;53(1):55-8.

37. Homesley HD, Bundy BN, Sedlis A, Adcock L. Radiation therapy versus pelvic node resection for carcinoma of the vulva with positive groin nodes. Obstet Gynecol 1986;68(6):733-40.

38. Mahner S, Jueckstock J, Hilpert F, Neuser P, Harter P, de Gregorio N, et al. Adjuvant therapy in lymph nodepositive vulvar cancer: The AGO-CaRE-1 study. J Natl Cancer Inst 2015;107(3):426.

39. van der Velden J, Pleunis N, Barlow E, Zijlmans H, de Hullu J, Hacker NF, et al. Radiotherapy is not indicated in patients with vulvar squamous cell carcinoma and only one occult intracapsular groin node metastasis. Gynecol Oncol 2021;160(1):128-33.

40. Parthasarathy A, Cheung MK, Osann K, Husain A, Teng NN, Berek JS, et al. The benefit of adjuvant radiation therapy in single-node-positive squamous cell vulvar carcinoma. Gynecol Oncol 2006;103(3):1095-9.

41. Serre E, Raimond E, Diguisto C, Bendifallah S, Body $\mathrm{G}$, Touboul C, et al. Inguino-femoral radiotherapy in vulvar squamous cell carcinoma: Clues to revised indications in patients with only one intracapsular lymph 
node metastasis. Acta Oncol 2020;59(5):518-24.

42. Gill BS, Bernard ME, Lin JF, Balasubramani GK, Rajagopalan MS, Sukumvanich P, et al. Impact of adjuvant chemotherapy with radiation for node-positive vulvar cancer: A National Cancer Data Base (NCDB) analysis. Gynecol Oncol 2015;137(3):365-72.

43. Gaffney DK, King B, Viswanathan AN, Barkati M, Beriwal S, Eifel P, et al. Consensus recommendations for radiation therapy contouring and treatment of vulvar carcinoma. Int J Radiat Oncol Biol Phys 2016;95(4):1191-200.

44. Gadducci A, Cionini L, Romanini A, Fanucchi A, Genazzani AR. Old and new perspectives in the management of high-risk, locally advanced or recurrent, and metastatic vulvar cancer. Crit Rev Oncol Hematol 2006;60(3):227-41.

45. Boronow RC. Combined therapy as an alternative to exenteration for locally advanced vulvo-vaginal cancer: Rationale and results. Cancer 1982;49:1085-91.

46. Bartelink H, Roelofsen F, Eschwege F, Rougier P, Bosset JF, Gonzalez DG, et al. Concomitant radiotherapy and chemotherapy is superior to radiotherapy alone in the treatment of locally advanced anal cancer: Results of a phase III randomized trial of the European organization for research and treatment of cancer radiotherapy and gastrointestinal cooperative groups. J Clin Oncol 1997;15(5):2040-9.

47. Morris M, Eifel PJ, Lu J, Grigsby PW, Levenback C, Stevens RE, et al. Pelvic radiation with concurrent chemotherapy compared with pelvic and para-aortic radiation for high-risk cervical cancer. N Engl J Med 1999;340:1137-43.

48. Moore DH, Thomas GM, Montana GS, Saxer A, Gallup DG, Olt G. Preoperative chemoradiation for advanced vulvar cancer: A phase II study of the Gynecologic Oncology Group. Int J Radiat Oncol Biol Phys 1998;42(5):79-85.

49. Montana GS, Thomas GM, Moore DH, Saxer A, Mangan CE, Lentz SS, et al. Preoperative chemo-radiation for carcinoma of the vulva with N2/N3 nodes: A gynecologic oncology group study. Int J Radiat Oncol Biol Phys 2000;48(4):1007-13.

50. Moore DH, Ali S, Koh WJ, Michael H, Barnes MN, McCourt CK, et al. A phase II trial of radiation therapy and weekly cisplatin chemotherapy for the treatment of locally-advanced squamous cell carcinoma of the vulva: A gynecologic oncology group study. Gynecol Oncol 2012;124(3):529-33.

51. Natesan D, Hong JC, Foote J, Sosa JA, Havrilesky L, Chino J. Primary versus preoperative radiation for locally advanced vulvar cancer. Int J Gynecol Cancer 2017;27(4):794-804.

52. Kim CH, Olson AC, Kim H, Beriwal S. Contouring inguinal and femoral nodes; how much margin is needed around the vessels? Pract Radiat Oncol 2012;2(4):2748.

53. Chapman BV, Gill BS, Viswanathan AN, Balasubramani GK, Sukumvanich P, Beriwal S. Adjuvant radiation therapy for margin-positive vulvar squamous cell carcinoma: Defining the ideal dose-response using the national cancer data base. Int J Radiat Oncol Biol Phys 2017;97(1):107-17.

54. D'Souza DP, Rumble RB, Fyles A, Yaremko B, Warde P, Members of the IIEP. Intensity-modulated radiotherapy in the treatment of gynaecological cancers. Clin Oncol (R Coll Radiol) 2012;24(7):499-507.

55. Beriwal S, Heron DE, Kim H, King G, Shogan J, Bahri $S$, et al. Intensity-modulated radiotherapy for the treatment of vulvar carcinoma: A comparative dosimetric study with early clinical outcome. Int J Radiat Oncol Biol Phys 2006;64(5):1395-400.

56. Rao YJ, Chundury A, Schwarz JK, Hassanzadeh C, DeWees T, Mullen D, et al. Intensity modulated radiation therapy for squamous cell carcinoma of the vulva: Treatment technique and outcomes. Adv Radiat Oncol 2017;2(2):148-58.

57. Beriwal S, Shukla G, Shinde A, Heron DE, Kelley JL, Edwards RP, et al. Preoperative intensity modulated radiation therapy and chemotherapy for locally advanced vulvar carcinoma: analysis of pattern of relapse. Int J Radiat Oncol Biol Phys 2013;85(5):1269-74.

58. Beriwal S, Coon D, Heron DE, Kelley JL, Edwards $\mathrm{RP}$, Sukumvanich $\mathrm{P}$, et al. Preoperative intensitymodulated radiotherapy and chemotherapy for locally advanced vulvar carcinoma. Gynecol Oncol 2008;109(2):291-5.

59. Bloemers MC, Portelance L, Ruo R, Parker W, Souhami L. A dosimetric evaluation of dose escalation for the radical treatment of locally advanced vulvar cancer by intensity-modulated radiation therapy. Med Dosim 2012;37(3):310-3. 